\title{
Composición Química del Aceite Esencial de las Hojas de Toronjil (Melissa officinalis L.)
}

\section{Diofanor Acevedo, Mario Navarro y Piedad Montero}

Universidad de Cartagena, Facultad de Ingeniería, Programa de ingeniería de Alimentos, Av. el Consulado, Calle 30 No. 48-152. Cartagena, Bolívar-Colombia (e-mail: diofanor3000@gmail.com)

* Autor a quien debe ser dirigida la correspondencia

Recibido Ene. 09, 2013; Aceptado Feb. 18, 2013; Versión final recibida Abr. 01, 2013

\section{Resumen}

Se estableció la composición química del toronjil (Melissa officinalis L.) cultivado en Coloso, Departamento de Sucre en Colombia, mediante la técnica de cromatografía de gases acoplada a espectrometría de masas. Esto fue realizado para determinar la variabilidad en la composición química producida por las condiciones climáticas que presenta la región. El toronjil se usa en esta comunidad en bebidas aromáticas y como tranquilizante natural considerando sus múltiples propiedades antioxidantes, microbiológicas, conservantes y aromatizantes de alimentos. Se identificó eugenol como el compuesto mayoritario con $45.47 \%$, seguido del cariofileno, a-cariofileno, 3-metil-4-isopropil fenol, germacreno D, con $40.77 \%, 2.94 \%$, $1.52 \%$ y $1.17 \%$ respectivamente. De acuerdo a los resultados, el aceite esencial de toronjil está de acuerdo con el quimiotipo eugenol.

Palabras clave: Melissa officinalis L, eugenol, cromatografía de gases, espectrometría de masas.

\section{Chemical Composition of the Essential Oil from Lemon Balm Leaves (Melissa officinalis L.)}

\begin{abstract}
Chemical composition of Lemon balm (Melissa officinalis L.) grown in Coloso, Department of Sucre in Colombia, using the technique of gas chromatography coupled with mass spectrometry was determined. This was done to establish the variability in the chemical composition caused by weather conditions presented region. Lemon balm is used in this community in aromatic beverages and as a natural tranquilizer, considering its several antioxidant, microbiological, preserving and aromatic properties. eugenol was determined as the major component $45.47 \%$, followed caryophyllene, a-caryophyllene, 3-methyl-4isopropyl phenol, germacrene $\mathrm{D}$, with $40.77 \%, 2.94 \%, 1.52 \%$ and $1.17 \%$ respectively. According to the results, the essential oil of lemon balm agrees with the eugenol chemotype.
\end{abstract}




\section{INTRODUCCIÓN}

En los últimos años se ha evidenciado un extraordinario auge de la química de los productos naturales en el ámbito mundial. Entre los tres grupos de productos de origen botánico que con mayor probabilidad tendrán el impacto más notable en la protección de plantas en la próxima década se encuentran los aceites esenciales y sus constituyentes, provenientes de diferentes especies vegetales (Esquivel \& Vargas, 2007; Isman, 2006; Simándi et al., 1998). Los aceites esenciales son usados como agentes carminativos, estimulantes, diuréticos y antirreumáticos; algunos poseen propiedades insecticidas, antifúngicas y antibacterianas frente a microorganismos patógenos y han sido considerados como ingredientes activos en algunos plaguicidas botánicos, debido a su eficacia frente a un número considerable de plagas, su toxicidad mínima en mamíferos y su disponibilidad general (Acevedo et al., 2007; Isman, 2006).

Melissa officinalis $L$. pertenece a la familia Lamiaceae, es conocida en nuestro país como toronjil y en otros como melissen blat o lemon balm por su olor a limón, es una planta medicinal muy estudiada para la que se describen como principales compuestos los ácidos hydroxycinámicos y los aceites esenciales, donde los mayores constituyentes son los terpenoides como el citral, citronelal, geraniol, nerol y linalol (Esquivel \& Vargas, 2007). Otros compuestos de interés presentes en esta especie son flavonoides y taninos (Stashenko et al., 2006; WHO, 2002). Se han demostrado variaciones cualitativas de este por factores genéticos y variaciones cualitativas debidas a las condiciones ambientales, duración del día y composición del suelo (Sadraei, 2003; Contreras et al., 2003). El rendimiento de la extracción y la composición química del aceite esencial de $M$. officinalis $L$, depende del microambiente en el cual crece la planta y del periodo en el cual son cosechadas las hojas (Saeb y Gholamrezaee, 2012).

En Colombia se han identificado aproximadamente 23 géneros y cerca de 205 especies de plantas de la familia Labiatae (Pérez et al., 2008). La infusión de las hojas de las plantas de esta familia como Hyptis capitata y Salvia officinalis se usan en la medicina tradicional colombiana, como antisépticas, antiinflamatorias, antiespasmódicas y analgésicos. (Fonnegra, 2006). Diferentes estudios han mostrado actividad antimicótica in vitro de aceites esenciales de plantas de la familia Labiatae como M. officinalis, $H$. ovalifolia, Mentha piperita y Ocimum basilicum contra aislados clínicos de Trichophyton rubrum, mentagrophytes y Microsporum canis (Bozin et al., 2006; Iscan, 2002). En la extracción de aceites esenciales es importante conocer y describir el tipo de material vegetal que se usa en el proceso (Carhuapoma, 2006; Arcila-Lozano et al., 2004). Por tanto, si existen variedades intrínsecas en organismos vegetales de la misma especie, estos se deben clasificar y diferenciar para evitar cambios en la composición del producto final, ajenos al proceso mismo de extracción, manipulación de material y tipo de análisis (Valenzuela et al., 2003; Acevedo et al., 2007; Carhuapoma, M. 2006). El análisis de quimiotipos se basa en la diferenciación de metabolitos secundarios y se aplica, no sólo a organismos vegetales, sino también a líquenes y algunos microorganismos. Por lo general, al analizar un aceite esencial se utiliza la técnica de cromatografía de gases acoplada a espectrometría de masas (CG-EM), como técnica de identificación y, para este caso, caracterización de quimiotipos con base en los compuestos mayoritarios (Simándi et al., 1998). Además, la importancia de los aceites esenciales y de sus componentes químicos, se basa en su implementación como aditivos antibacteriales en productos alimenticios (Burt 2007; Esquivel \& Vargas, 2007) y en farmacología (Acevedo et al., 2007); también el valor económico y la aplicabilidad industrial de las esencias se relacionan de manera directa con su composición química, que a su vez determina todas las propiedades (fisicoquímicas, organolépticas) (Albado et al., 2001).

El objetivo del presente trabajo fue determinar la composición química del aceite esencial extraído de las hojas de toronjil (M. officinalis $L$ ) procedentes del municipio de Coloso (Sucre-Colombia), por medio de la técnica de cromatografía de gases acoplada a espectrometría de masas.

\section{MATERIALES Y MÉTODOS}

\section{Obtención de materia prima}

Las hojas de $M$. officinalis $L$, fueron recogidas de parcelas artesanales ubicadas en el municipio de Coloso, Departamento de Sucre, Colombia. El material se recolectó de forma manual, en la zona es comúnmente conocido como toronjil silvestre, que es una planta herbácea anual, de la familia de las Labiadas, con muchos tallos rectos de cuatro a seis decímetros de altura, hojas pecioladas, ovales, arrugadas, dentadas y olorosas, flores blancas en verticilos axilares, y fruto seco, capsular, con cuatro semillas menudas (Allahverdiyev et al., 2004). 


\section{Extracción del aceite esencial}

El modo de extracción que se utilizo fue hidrodestilación con trampa de Clevenger, el procedimiento consistió en picar finamente $1000 \mathrm{gr}$ de material fresco de hojas de $M$. officinalis $L$, depositarlo en un recipiente de acero inoxidable, al que se le añadieron $2000 \mathrm{ml}$ de agua destilada, con un tiempo de extracción de 120-180 minutos. (Stashenko et al., 2006; WHO, 2002).

\section{Caracterización química del aceite esencial}

Los procedimientos para determinar la composición química del aceite esencial de toronjil, se realizaron bajo la técnica de cromatografía de gases acoplada a espectrometría de masas (CG-EM).

Se necesitaron $50 \mu \mathrm{l}$ de aceite esencial para el análisis cromatográfico y se aforo a $450 \mu \mathrm{l}$ con diclorometano. La mezcla se realizó en un matraz aforado de $1.5 \mathrm{ml}$ y después se transfirió a un vial para cromatografía de gases de $2 \mathrm{ml}$. Este análisis se llevó a cabo en un Cromatografo de gases - Agilent Technologies 7890A, acoplado a un espectrómetro de masas Agilent Technologies 5975C equipado con un puerto de inyección Split/splitlles (relación 1:50), un inyector automático Agilent 7863 y un sistema de datos HP Chem Station.

Para la separación de las mezclas se utilizó una columna capilar DB-5MS( $\mathrm{J} \& W$ Scientific, Folsom, EE.UU.) con fase estacionaria de $5 \%$ fenil-polimetilsiloxano $(60 \mathrm{~m} \times 0,25 \mathrm{~mm}$, D.I. $\times 0,25 \mu \mathrm{m}$, df). La temperatura del horno se programó desde $45^{\circ} \mathrm{C}(5 \mathrm{~min})$ hasta $150^{\circ} \mathrm{C}(2 \mathrm{~min})$ a $4^{\circ} \mathrm{C} / \mathrm{min}$, luego se incrementó hasta $250^{\circ} \mathrm{C}(5 \mathrm{~min})$ a $5^{\circ} \mathrm{C} / \mathrm{min}$, finalmente, la temperatura aumentó a $10^{\circ} \mathrm{C} / \mathrm{min}$, hasta alcanzar $275^{\circ} \mathrm{C}(15$ min). Las temperaturas de la cámara de ionización y de la línea de transferencia fueron de 230 y $285^{\circ} \mathrm{C}$, respectivamente. El gas de arrastre empleado fue helio (99.995\%). Los espectros de masas y corriente iónicas reconstruidas se obtuvieron por medio de barrido automático de frecuencia ( full scan), a 4.75 scan $\mathrm{s}-1$, en el rango de masas $\mathrm{m} / \mathrm{z}$ 30-450.

Para el análisis por la columna polar se empleó un cromatógrafo de gases Agilent Technologies 6890 Network GC System, acoplado a un detector selectivo de masas Agilent Technologies 5975, equipado con un puerto de inyección split/splitless $\left(250^{\circ} \mathrm{C}\right.$, split 1:14) y un sistema de datos HP ChemStation D.02.00.275. El proceso de separación se llevó a cabo en una columna capilar DB-WAX ( $\mathrm{J} \& W$ Scientific, Folsom, CA, EE.UU.) con fase estacionaria entrecruzada e inmovilizada de polietilenglicol $(60 \mathrm{~m} \times 0,25 \mathrm{~mm}$, D.I. $\times 0,25$ $\mu \mathrm{m}, \mathrm{df})$. La temperatura del horno se programó desde $45^{\circ} \mathrm{C}(10 \mathrm{~min})$ hasta $220^{\circ} \mathrm{C}(30 \mathrm{~min})$ a $3^{\circ} \mathrm{C} / \mathrm{min}$. Las temperaturas de la cámara de ionización y de la línea de transferencia fueron de 230 y $250^{\circ} \mathrm{C}$, respectivamente. La identificación de los compuestos se realizó mediante la comparación de los tiempos de retención y de los patrones de fragmentación representados en los espectros de masas experimentales con los encontrados en las bases de datos o librerías (Data base INIST08.L, DatabaselW8.I). Stashenko et al., 2006; WHO, 2002).

\section{ANÁLISIS DE LOS RESULTADOS}

Los resultados que se obtuvieron por GC-MS del aceite esencial de Toronjil, revelan los principales componentes químicos, destacando el Eugenol con $45,47 \%$, seguido del cariofileno, $\alpha$-cariofileno, 3- metil-4isopropil fenol, germacreno $\mathrm{D}$, con $40,77 \%, 2,94 \%, 1,52 \%, 1,17 \%$ respectivamente, que representan más del $90 \%$ del total registrado(tabla1). Los datos arrojados muestran una gran diferencia en la composición química de esta especie que se cultiva en Coloso ya que su principal componente no se encuentra relacionado en la bibliografía encontrada. Ya que el aceite que contienen las hojas de toronjil es un aceite que está compuesto por diferentes aldehídos y alcoholes sesquiterpénicos: citral a y b, citronelal, geraniol y linalol. Además, se encuentran taninos, ácidos triterpénicos, marrubina y saponinas, compuestos amargos y flavonoides. Toda la planta tiene un agradable aroma y sabor que recuerda al limón (Allahverdiyev et al., 2004), en esta especie estos componentes fueron encontrados en menor proporción. Las diferencias presentadas en su composición, pueden deber a varios factores; en ese sentido Bandoni et al., (2009) expresaron que es casi imposible lograr dos aceites esenciales idénticos. Como resultado del grado de sensibilidad analítica con que se trabaje, siempre se podrá encontrar alguna diferencia entre dos partidas de un mismo aceite esencial, por la época de cosecha, el año, el método de extracción, las condiciones de almacenamiento. De igual forma, variables como las condiciones geobotánicas, tipo de suelo, época de recolección y edad de la planta (Bandoni et al., 2009; Contreras et al., 2003; Albado et al., 2001), entre otros. 
Tabla 1: Componentes del aceite esencial de toronjil (M. officinalis $L$ ) identificados por GS-MS

\begin{tabular}{|c|c|c|c|}
\hline $\operatorname{Tr}(\min )$ & Compuesto & familia química & Área relativa (\%) \\
\hline 21,69 & 3- metil-4-isopropil fenol & Compuesto oxigenado & 1,52 \\
\hline 23,33 & Eugenol & Compuesto oxigenado & 45,47 \\
\hline 24,97 & Cariofileno & Sesquiterpeno bicíclico & 40,77 \\
\hline 25,76 & a-cariofileno & Sesquiterpeno bicíclico & 2,94 \\
\hline 26,44 & germacreno D & Compuesto terpénico & 1,17 \\
\hline
\end{tabular}

El quimiotipo de esta especie pertenece al Eugenol y Cariofileno por ser los mayores componentes mayoritarios ambos sobrepasan el $40 \%$ de su contenido en el aceite. La importancia de esta planta radica en que su principal componente tiene muchas propiedades entre las que se destacan fundamentalmente sus propiedades antiagregantes, antiedemicas, antimovitivas, carminativas, antiácidas, antioxidantes, gastroprotectoras, gastrorregenerativas, bactericidas, antifúngicas, antisépticas, antivirales, herbicidas, pesticidas, insecticidas, insectifugas, vermifugas, anestésicas, sadativas, fragantes y aromatizantes (Huerta, 2007). La industria alimentaria lo utiliza para dar aromas a comidas preparadas, bebidas con alcohol, y otros refrescos, además eugenol provenientes de clavo y tomillo causan la desintegración de la membrana de $E$. coli y S. typhiurium. El eugenol (componente mayoritario del aceite de clavo) y el cinamaldehido (componente de la canela) actúan inhibiendo la producción de enzimas intracelulares, tales como amilasas y proteasas, lo que provoca el deterioro de la pared y un alto grado de lisis celular. (Mitsch et al., 2004, Huerta, 2007).

\section{CONCLUSIONES}

La composición química que presenta el aceite esencial de toronjil cultivado en Coloso Sucre (Colombia) pertenece al quimiotipo Eugenol algo que no es muy común en esta especie cultivada en otras partes. Este compuesto permite dar gran valor agregado a esta planta, por su alto porcentaje en masa ya que sus principales componentes se encuentran en altos porcentajes, lo que convierte a esta especie en una posible fuente natural de estos compuestos como lo son el eugenol y el cariofileno, estos dos compuestos le otorgan múltiples propiedades antioxidantes, microbiológicas y conservantes y aromatizantes de alimentos, así también como su uso en muchas otras industrias.

\section{REFERENCIAS}

Acevedo A. y otros seis autores, Composición y capacidad antioxidante de especies aromáticas y medicinales con alto contenido de TIMOL Y CARVACROL, Scientia et Technica, 13(33), 125-128 (2007).

Allahverdiyev, A. y otros cuatro autores, Antiviral activity of the volatile oils of Melissa officinalis L. against Herpes simplex virus type-2, Phytomedicine, 11(7-8), 657-61 (2004).

Albado, E., Saez, G. y Grabiel, S., Composición química y actividad antibacteriana del aceite esencial del Origanum vulgare (orégano). Revista Medica Herediana, 12(1), 17-19 (2001).

Arcila-Lozano, C. y otro tres autores, Orégano: Propiedades, composición y actividad biológica de sus componentes, ALAN, 54(1), 100-111 (2004).

Bandoni, A. y otros tres autores, ¿Son realmente útiles los aceites esenciales?, BLACPMA, 8(5), 317-22 (2009).

Bozin B. y otros tres autores, Characterization of the volatile composition of essential oils of some lamiaceae spices and the antimicrobial and antioxidant activities of the entire oils, J Agric Food Chem., 54(5), 1822-8 (2006).

Burt, S.A., Antibacterial activity of essential oils: potential applications in food, PhD thesis, Institute for Risk Assessment Sciences, Division of Veterinary Public Health, Utrecht University, Utrecht, Netherlands (2007).

Carhuapoma, M., Estudio de la composición química y actividad antioxidante del aceite esencial de Luma chequen (Molina) A. Gray "arrayán". Tesis de maestría, Facultad de farmacia y bioquímica, Universidad Nacional Mayor de San Marcos, Lima, Perú (2006).

Contreras, N., Martínez, J. y Stashenko, E., Determinación de la actividad antioxidante in vitro de los aceites volátiles de cuatro plantas de uso tradicional mediante la medición de la peroxidación lipídica de aceite. Scientia et technica, 12(30), 365-369 (2006). 
Copping, Lg. \& Duke So., Natural products that have been used commercially as crop protection agents. Pest Manag Sci., 63(6), 524-554 (2007).

Esquivel, A. \& Vargas P., Uso de aceites esenciales extraídos por medio de fluidos supercríticos para la elaboración de alimentos funcionales. Tecnología en Marcha. 20(4), 41-50 (2007).

Fonnegra, R., Plantas medicinales aprobadas en Colombia, 2da edición, 193-196, editorial Universidad de Antioquia, Medellín, Colombia (2006).

Huerta, B., Aceites esenciales en el control de las patologías aviares (2008), http://www.wpsaaeca.es/aeca_imgs_docs/wpsa1177323612a.pdf

Iscan, G. y otros cuatro autores, Antimicrobial Screening of Mentha piperita Essential Oils, J Agric Food Chem., 50(14), 3943-3946 (2002).

Isman, M., Botanical insecticides, deterrents, and repellents in modern agriculture and an increasingly regulated world, Annual Review of Entomology, 51, 45-66 (2006).

Mitsch P. y otros cuatro autores, The effect of two different blends of essential oil components on the proliferation of Clostridium perfringens in the intestines of broiler chickens, Poultry Science, 83(4), 669-675 (2004).

Saeb, K. \& Gholamrezaee, G., Variation of essential oil composition of Melissa officinalis L. leaves during different stages of plant growth, Asian Pacific Journal of Tropical Biomedicine, 2(1), 547-549 (2012).

Sadraei H., Ghamadi A. y Malekshahi K., Effect of essential oil of M. officinalis and citral on ratileon contraction, Fitoterapia, 74, 445-52 (2003).

Simándi, B. y otros seis autores, Supercritical carbon dioxide extraction and fractionation of oregano oleoresin. Food Research International, 31(10), 723-728 (1998).

Stashenko, E., Jaramillo, B. y Martínez, J., Comparación de la composición química y de la actividad antioxidante in vitro de los metabolitos secundarios volátiles de plantas de la familia Verbenaceae, Rev. Acad. Colomb. Cienc., 27(105): 579-597 (2003).

Valenzuela, A., Sanhueza, J. y Nieto, S. Natural antioxidants in functional foods: from food safety to health benefits. Grasas y Aceites, 54(3), 295-303 (2003).

WHO, Monographs on Selected medicinal plants, Vol. 2, 180-187, Ginebra: World Health Organization (2002). 
Composición Química del Aceite Esencial de las Hojas de Toronjil (Melissa officinalis L.) Acevedo 